\title{
Clonorchis sinensis granulin: identification, immunolocalization, and function in promoting the metastasis of cholangiocarcinoma and hepatocellular carcinoma
}

\author{
Caiqin Wang ${ }^{1,2,3 \dagger}$, Huali Lei ${ }^{1,2,3,4+}$, Yanli Tian ${ }^{1,2,3}$, Mei Shang ${ }^{1,2,3}$, Yinjuan Wu ${ }^{1,2,3}$, Ye Li ${ }^{1,2,3}$, Lu Zhao ${ }^{1,2,3}$, \\ Mengchen Shi ${ }^{1,2,3}$, Xin Tang ${ }^{1,2,3}$, Tingjin Chen ${ }^{1,2,3}$, Zhiyue Lv ${ }^{1,2,3}$, Yan Huang ${ }^{1,2,3}$, Xiaoping Tang ${ }^{4}$, \\ Xinbing $\mathrm{Yu}^{1,2,3^{*}}$ and Xuerong $\mathrm{Li}^{1,2,3^{*}}$
}

\begin{abstract}
Background: Long-term infections by Clonorchis sinensis are associated with cholangitis, cholecystitis, liver fibrosis, cirrhosis, and even liver cancer. Molecules from the worm play vital roles in disease progress. In the present study, we identified and explored molecular characterization of C. sinensis granulin (CSGRN), a growth factor-like protein from C. sinensis excretory/secretory products (CSESPS).
\end{abstract}

Methods: The encoding sequence and conserved domains of CsGRN were identified and analysed by bioinformatics tools. Recombinant CsGRN (rCsGRN) protein was expressed in Escherichia coli BL21 (DE3). The localisation of CsGRN in adult worms and Balb/c mice infected with C. sinensis was investigated by immunofluorescence and immunohistochemistry, respectively. Stable CsGRN-overexpressed cell lines of hepatoma cells (PLC-GRN cells) and cholangiocarcinoma cells (RBE-GRN cells) were constructed by transfection of eukaryotic expression plasmid of pEGFP-C1-CsGRN. The effects on cell migration and invasion of CsGRN were assessed through the wound-healing assay and transwell assay. The levels of matrix metalloproteinase 2 and 9 (MMP2 and MMP9) in PLC-GRN or RBE-GRN cells were detected by real-time PCR (qRT-PCR). The levels of E-cadherin, vimentin, $\mathrm{N}$-cadherin, zona occludens proteins (ZO-1), $\beta$-catenin, phosphorylated ERK ( $p$-ERK) and phosphorylated AKT ( $p$-AKT) were analysed by Western blotting.

Results: CsGRN, including the conserved GRN domains, was confirmed to be a member of the granulin family. CsGRN was identified as an ingredient of CsESPs. CsGRN was localised in the tegument and testes of the adult worm. Furthermore, it appeared in the cytoplasm of hepatocytes and biliary epithelium cells from infected Balb/c mouse. The enhancement of cell migration and invasion of PLC-GRN and RBE-GRN cells were observed. In addition, CsGRN upregulated the levels of vimentin, N-cadherin, $\beta$-catenin, MMP2 and MMP9, while it downregulated the level of ZO-1 in PLC-GRN/RBE-GRN cells. In total proteins of liver tissue from rCsGRN immunised Balb/c mice, vimentin level decreased, while E-cadherin level increased when compared with the control groups. Meanwhile, the levels of p-ERK reached a peak at 4 weeks post immunisation and the level of p-AKT did at 2 weeks after immunisation.

(Continued on next page)

\footnotetext{
* Correspondence: yuhxteam@163.com; xuerong2@mail.sysu.edu.cn

${ }^{\dagger}$ Equal contributors

${ }^{1}$ Department of Parasitology, Zhongshan School of Medicine, Sun Yat-sen

University, Guangzhou 510080, People's Republic of China

Full list of author information is available at the end of the article
} 
(Continued from previous page)

Conclusions: The encoding sequence and molecular characteristics of CsGRN were identified. As a member of granulin superfamily, CsGRN induced mesenchymal characteristics of PLC and RBE cells and was found to regulate the activities of the downstream molecules of the ERK and PI3K/AKT signalling pathways, which could contribute to the enhancement of cell migration and invasion.

Keywords: Clonorchis sinensis, Granulin, Cholangicarcinoma, Hepatocellular carcinoma, Immunolocalization, Cell migration

\section{Background}

Clonorchis sinensis, a human liver fluke, is a major foodborne parasite in China [1]. Long-term infections can lead to liver fibrosis, cirrhosis and even carcinogenesis [2, 3]. Some epidemiological studies and clinical researchers have shown that the incidence rate of primary hepatocellular carcinoma (HCC) is much higher in patients infected with C. sinensis than in non-infected patients [4-6].

A homologue of granulin from $C$. sinensis (CsGRN) was identified in our previous studies, which was predicted to be a component of excretory/secretory products (ESPs) [7, 8]. Granulins are a family of secreted, glycosylated peptides that are cleaved from a single precursor protein with 7.5 repeats of a highly conserved 12cysteine granulin/epithelin motif [9]. As independent growth factors, granulin family members are excessively expressed in various tumour tissues and are important in normal development, wound healing, and tumorigenesis [10]. Overexpression of progranulin (PGRN), which is a 60 to $90 \mathrm{kDa}$ glycoprotein containing seven tandemly repeated granulin motifs in mammals, is linked to tumorigenesis in numerous human tissues, including liver. It is also associated with an aggressive and invasive tumour phenotype [11]. The ov-grn-1, a granulin among the ESPs of Opisthorchis viverrini, may establish a tumorigenic environment that ultimately manifests as cholangiocarcinoma (CCA) $[12,13]$. FhGLM, a granulinlike molecule in Fasciola hepatica that is likely to be secreted through a nonclassical pathway, might exert a proliferative action on host cells during fascioliasis [14].

ESPs of C. sinensis (CsESPs) were reported as one of the most important factors for pathogenesis [15]. Our previously studies showed that some molecules from CsESPs could cause obvious apoptotic inhibition, and promote proliferation and migration of human $\mathrm{HCC}$ cells, which might exacerbate the process of HCC patients combined with $C$. sinensis infection [16, 17].

Given the close phylogenetic relationship with liver flukes and topologically similarity to both ov-grn- 1 and PGRN, we proposed that CsGRN may have a similar biological function of other granulin superfamily members as a growth factor. In the present study, the molecular characteristics of CsGRN and its potential roles in the pathogenesis of clonorchiasis were investigated.

\section{Methods}

Parasite and preparation of CsESPs

Adult worms of $C$. sinensis were isolated from the bile ducts of infective cats. After washing procedures, the adult worms were used for tissue sections preparation or total RNA extraction. Adult worms were also cultivated to collect CsESPs and eggs, according to the method described previously [18].

\section{Bioinformatics analysis of the CsGRN sequence}

The mRNA sequence annotated with granulin (CsGRN) was selected from our $C$. sinensis cDNA plasmid library and was identified by DNA sequencing. The domains, physicochemical properties and some structures of the translated amino acid sequence were predicted with proteomics tools at NCBI (http://www.ncbi.nlm.nih.gov) and ExPaSy website (http://www.expasy.org/), and the disulphide bonds were analysed through the website (http://scratch.proteomics.ics.uci.edu/). The multiple alignments of sequences with homologues from human and helminths were carried out by Vector NTI software, and the phylogenetic tree was constructed with corresponding sequences from 14 other species using the software MEGA version 6.0 [19].

\section{Gene cloning, expression and purification of recombinant CsGRN}

The ORF of CsGRN (GenBank KY855531) was 714 bp and the specific primers were as follows: forward $5^{\prime}-\mathrm{C}$ GC GGA TCC TGT AAA TAT AAC CAG ACT TG-3' (BamH I) (Thermo Scientific, Waltham, USA), Reverse: 5'-TTA CTC GAG CGG AGC ACA GGT GTA GTG AT-3' (Xhol I) (Thermo Scientific). The underlined bases indicated restrictions sites. cDNA was synthesised from total RNA, which was isolated from frozen $C$. sinensis adult tissues. The amplification conditions were $94{ }^{\circ} \mathrm{C}$ for $1 \mathrm{~min}, 55^{\circ} \mathrm{C}$ for $1 \mathrm{~min}$ and $72{ }^{\circ} \mathrm{C}$ for $1 \mathrm{~min}$ for 30 cycles, plus $72{ }^{\circ} \mathrm{C}$ for $10 \mathrm{~min}$. The purified PCR products were ligated into the pGEM-T-Easy vector (Promega, Madison, USA), followed by transformation into E. coli DH5 $\alpha$ (Promega). The resulting plasmid DNA was digested with the appropriate restriction enzymes, ligated into the pET-28a $(+)$ expression vector (Novagen, Darmstadt, Germany), and then transformed 
into E. coli BL21 (DE3) (Promega). Selected clones were grown and induced with $1 \mathrm{mM}$ isopropyl- $\beta$-d-thiogalactoside (IPTG, Sigma, Guangzhou, China) at $20{ }^{\circ} \mathrm{C}$ for 12 $18 \mathrm{~h}$. The bacterial cells were collected by centrifugation and were sonicated on ice. The supernatant was collected, and recombinant protein was purified using the His-Bind Purification Kit (Novagen). The lysates of purified protein were subjected to $12 \%$ sodium dodecyl sulfatepolyacrylamide gel electrophoresis (SDS-PAGE). The concentration of purified recombinant protein was determined by using the BCA protein assay kit (Novagen).

\section{Preparation of antiserum for CsESPs and CsGRN}

The recombinant CsGRN (rCsGRN) and CsESPs were emulsified with complete Freund' s adjuvant (Sigma-Aldrich, Guangzhou, China) and were subcutaneously immunised with $200 \mu \mathrm{g}$ of protein for each rat initially. Subsequently, each rat was given $100 \mu \mathrm{g}$ of protein (emulsified with equivalent incomplete Freund's adjuvant (Sigma-Aldrich) for three booster injections at 2-week intervals. Anti-CsGRN serum was collected every 2 weeks. Two weeks after the final boosting, the rats were sacrificed, and the sera were collected. Sera from naïve rats were also collected as a control. The antibody titers were determined by enzyme-linked immunosorbent assay (ELISA) and immunoblot analysis. For the production of mouse anti-CsGRN serum, $\mathrm{Balb} / \mathrm{c}$ mice were initially immunised with $100 \mu \mathrm{g}$ of purified $\mathrm{rCs}$ GRN followed by $50 \mu \mathrm{g}$ the next three times, as described in the above method. The mice were sacrificed, and the liver tissues and serum were collected every 2 weeks from the final boosting for 8 weeks. The liver tissues and serum from normal mice during the same periods were also collected as a control.

\section{Immunofluorescence assay (IFA)}

Freshly prepared $C$. sinensis adult worms were washed with phosphate buffer solution (PBS, $20 \mathrm{mM}$, pH 7.4) and then were fixed with $4 \%$ paraformaldehyde. The worms were dehydrated by a graded ethanol series, embedded in paraffin blocks and stored in a desiccator until use. Sections ( $4 \mu \mathrm{m}$ thickness) were mounted on slide glasses, deparaffinized, rehydrated and rinsed with PBS. The slides were incubated with rat anti-CsGRN or rat normal sera (1:50 dilutions) in PBS at room temperature for $2 \mathrm{~h}$ and then were washed with PBS three times. After incubation with $\mathrm{Cy}^{\text {rum }} 3$-conjugated anti-rat IgG (1:400 dilutions; Proteintech Group, Chicago, USA) for $2 \mathrm{~h}$, the slides were washed with PBS three times and were observed under a light/fluorescence microscope (Olympus BX63, Hatagaya, Japan).

\section{Immunohistochemical localisation of CsGRN}

Female Balb/c mice (6-8 weeks of age) in the C. sinensis group were intragastrically infected with metacercariae
(30 metacercariae per mouse). We determined the success of infection by stool examination. Five mice from each group were randomly selected and were sacrificed at 7, 30, 60, 90, 120 and 180 days postinfection (uninfected mice in same terms were used as controls). The liver tissues were extracted, and tissue sections were prepared. These samples were fixed and cut by a microtome of $4 \mu \mathrm{m}$ sections. The sections were incubated with mouse anti-CsGRN serum or mouse naïve serum (1:50 dilutions) overnight at $4{ }^{\circ} \mathrm{C}$ after being dewaxed in $\mathrm{xy}$ lene, dehydrated in ethanol, and blocked with normal goat serum. The sections were washed with PBST $(0.1 \%$ Tween-20 in PBS) and were incubated with horseradish peroxidase-conjugated goat anti-mouse IgG (1:400 dilutions; Proteintech Group) for $1 \mathrm{~h}$. The sections were rinsed with $\mathrm{PBS}$ for $15 \mathrm{~min}$, after which the slides were developed with diaminobenzidine (DAB). Next, the sections were counterstained with Mayer's haematoxylin, dehydrated, cleared in xylene and mounted in PermountH. Images were captured with a microscope (Olympus BX51, Hatagaya, Japan).

\section{Cells culture, construction of the eukaryotic expression plasmid pEGFP-C1-CsGRN and generation of stable cell line}

RBE (human cholangiocarcinoma cell line, ATCC), and PLC (human hepatocarcinoma cell line, ATCC) were maintained as specified by ATCC protocols. RBE cells were cultured in RPMI 1640 (Gibco, Carlsbad, USA) while PLC was routinely maintained in DMEM medium (Gibco). These cell lines were supplemented with $10 \%$ fetal bovine serum (FBS, Gibco) and penicillinstreptomycin (100 units $/ \mathrm{ml}$ ) in $5 \% \mathrm{CO}_{2}$ at $37{ }^{\circ} \mathrm{C}$.

Standard molecular biology techniques were used for the construction of the pEGFP-C1-CsGRN recombinant plasmid. The PCR product of the CsGRN fragment was cut and inserted between the Xhol I (Thermo Scientific) and EcoR I (Thermo Scientific) restriction sites in the pEGFP-C1 vector (Promega). The forward and reverse primers (restriction sites are underlined) used to amplify this fragment included forward 5'-GCG CCT CGA GTG TAA ATA TAA CCA GAC-3' (Xhol I) and reserve 5'-ATA AGG ATC CCG GAG CAC AGG TGT AG-3' $(E c o R$ I) respectively, based on the following conditions: $30 \mathrm{~s}$ denaturation at $94^{\circ} \mathrm{C}, 30 \mathrm{~s}$ annealing at $60^{\circ} \mathrm{C}$, and 1 min extension at $72{ }^{\circ} \mathrm{C}$ for 30 cycles, plus $72{ }^{\circ} \mathrm{C}$ for $10 \mathrm{~min}$. Cells $\left(1 \times 10^{6}\right)$ plated in a 6-well cell culture cluster were transfected with either $0.8 \mu \mathrm{g}$ of pGFP-C1 or pGFP-C1-CsGRN using lipofectamine 2000 (Invitrogen, Carlsbad, USA) according to the manufacturer's instructions. Two days after transfection, the stable cell line selection was started using the optimal concentration of G418. The medium was changed every 2-3 days, and the cells were split when necessary. After 2-4 
weeks, all of the non-transfected cells disappeared, and isolated colonies began to appear. The selective overexpression of GFP or CsGRN cells was designated as PLCGFP/RBE-GFP cells and PLC-GRN/RBE-GRN cells, respectively. CsGRN overexpression in these cells was checked by a fluorescence microscope, fluorescence activated cell sorting (FACS) analysis, qRT-PCR analysis and Western blotting first incubated with mouse antiCsGRN sera (1:100 dilution).

\section{Cell migration and invasion assay}

To further confirm the role of CsGRN in human cancer progression, wound-healing assays were performed to evaluate the effect of CsGRN on cell migration as described previously [20]. The transfected PLC and RBE cells seeded in 6-well plates were grown to $80 \%$ confluence and were wounded by scratching with p200 pipette tips. Wounds were observed and photographed under a light microscope (Leica DMI3000B, Wetzlar, Germany) every $24 \mathrm{~h}$ for $72 \mathrm{~h}$. The distances between the parallel cell edges were measured at each time point using Image J software. For each well, three different fields along the scratch were analysed in triplicate. Cell motility was measured as the percentage of the cell migration distance, which was regarded as the initial scratch distance. To evaluate the effect of CsGRN on cell invasion, we performed transwell assays as described previously [21]. The PLC-GFP/RBE-GFP cells and PLC-GRN/RBE-GRN cells were suspended at $5.0 \times 10^{4}$ per insert with serumfree media and then were transferred to wells filled with a culture medium containing $10 \%$ FBS as a chemoattractant. After $24 \mathrm{~h}$ of incubation, non-invading cells on the top of the membrane were removed with a cotton swab. The migrated cells on the underside of the filter membrane were fixed and stained with $0.1 \%$ crystal violet. The number of migrated cells on the membrane was counted in five randomly selected microscopic fields, and the cells were photographed. The protocol used for the invasion assay was the same as that used for the migration assay, except that the transwell insert was coated with Matrigel (BD Biosciences, Heidelberg, Germany).

\section{Real-time reverse transcription PCR}

Total RNA of transfected cells was isolated using the Trizol reagent and was reverse transcribed to cDNA using ABM's 5× All-In-One RT Master Mix (Transgen, Beijing, China). For qRT-PCR, SYBR Premix Ex Taq (Takara, Dalian, China) was used according to the manufacturer's instructions. The primers were as follows: for CsGRN, forward $5^{\prime}$-CGC GGA TCC TGT AAA TAT AAC CAG ACT TG-3' and reverse 5' ${ }^{\prime}$-TTA CTC GAG CGG AGC ACA GGT GTA GTG AT-3'; for MMP2, forward 5'-TAC AGG ATC ATT GGC TAC ACA CC-
3' and reverse 5' -GGT CAC ATC GCT CCA GAC T-3' ;for MMP9, forward 5' -TGT ACC GCT ATG GTT ACA CTC G-3' and reverse 5'-GGC AGG GAC AGT TGC TTC T-3'; for human actin, forward 5'-GGC ACT CTT AGC CTT CCT TCC T-3' and reverse 5'-GCC AGA CAG CAC TGT GTT GGC GT-3'. Reactions were conducted under the following conditions: $95{ }^{\circ} \mathrm{C}$ for $30 \mathrm{~s}, 40$ cycles of $95{ }^{\circ} \mathrm{C}$ for $5 \mathrm{~s}$, and $60{ }^{\circ} \mathrm{C}$ for $20 \mathrm{~s}$. The melting curves were analysed automatically by a collection of the fluorescence signals, and the expression of mRNA was calculated and normalised using the $2^{-\Delta \Delta C t}$ method relative to actin with CFX96 software (Bio-Rad, Hercules, USA). Independent experiments were performed in triplicate.

\section{Western blotting analysis}

CsGRN $(5 \mu \mathrm{g})$ and CsESPs $(30 \mu \mathrm{g})$ were subjected to $12 \%$ SDS-PAGE and then were electro-transferred onto polyvinylidene difluoride (PVDF) membranes (Whatman, Maidstone, UK) at $100 \mathrm{~V}$ for $1 \mathrm{~h}$ in a Trans-Blot transfer (Bio-Rad). The membranes were blocked with $5 \%(\mathrm{w} / \mathrm{v})$ skimmed milk in PBS for $2 \mathrm{~h}$ at room temperature and then were probed with mouse anti-His tag monoclonal antibody (1:2000, Novagen), rat antiCsGRN serum (1:200), rat anti-CsESPs serum (1:200) and rat naïve serum $(1: 200)$ at $4{ }^{\circ} \mathrm{C}$ overnight, respectively. After washing with PBS, the membranes were successively incubated with HRP-conjugated rabbit anti-rat or rabbit anti-mouse IgG at 1:2000 dilution (Proteintech Group) at room temperature for $1 \mathrm{~h}$. The blots were visualised by enhanced chemiluminescence (ECL, Millipore, Billerica, USA). We also used Western blotting to detect the mechanism of cancer progression induced by CsGRN. In detail, the total proteins of transfection cells were extracted using radioimmunoprecipitation assay lysis buffer (RIPA, Beyotime, Shanghai, China) and then were electrotransferred onto PVDF membranes. The membranes were incubated at $4{ }^{\circ} \mathrm{C}$ overnight with Ecadherin $(1: 2,000)$, vimentin $(1: 2,000)$, N-cadherin (1:2,000), ZO-1 (1:2,000), $\beta$-catenin (1:2,000), or GAPDH $(1: 2,000)$ diluted in blocking solution. In addition, the membranes with total proteins from $\mathrm{Balb} / \mathrm{c}$ mice liver tissues were incubated at $4{ }^{\circ} \mathrm{C}$ overnight with E-cadherin (1:2,000), vimentin $(1: 2,000)$, $\mathrm{N}$-cadherin $(1: 2,000), \mathrm{ZO}-1$ $(1: 2,000), \quad \beta$-catenin $(1: 2,000), \quad p$-ERK $(1: 2,000)$, ERK $(1: 2,000)$, p-AKT $(1: 2,000)$ or GAPDH $(1: 2,000)$ diluted in blocking solution, all antibody were products from cell signaling technology (CST, Boston, USA).

\section{Statistical analysis}

Experimental data were obtained from three independent experiments with a similar pattern; data are expressed as the means \pm standard deviation. The Student's $t$-test and ANOVA were used to determine the 
statistical significance of the data obtained, and the means were compared between the groups using SPSS21.0 statistical software. A $P<0.05$ represented a statistically significant difference.

\section{Results}

\section{Sequence analysis}

The sequence of CsGRN encoding 238 amino acids was predicted with molecular weight $25.8 \mathrm{kDa}$, theoretical point isoelectric (PI) of 8.5. It contains two granulin domains from sequence released in the GenBank (GAA54205.1), which is presumed without N-terminal signal peptide. Multiple alignments of the sequences among GRN and nine members from other organisms (Fig. 1a) indicated that it belongs to the granulin family with a conservative domain with rich cysteine peptides. They share low sequence identity to each other except for $O$. viverrini. In addition, the sequence is abundant with cysteines being presumed to contain thirteen disulphide bonds. Moreover, the phylogenetic analysis (Fig. 1b) suggested that the granulin protein from $C$. sinensis has a very close relationship to $O$. viverrini. Furthermore, the evolution of the members from host and trematode or nematode may vary greatly.

\section{Cloning, expression and purification of rCsGRN}

The ORF of CsGRN was cloned into the pET-28a (+) expression vector, and the recombinant plasmids were confirmed by restriction enzyme identification and DNA sequencing (not shown). The one mM IPTG-induced rCsGRN was purified and analysed by SDS-PAGE, and the purified protein was obtained from the supernatant using the His-Bind Purification Kit (Fig. 2a).

\section{Identification of CsGRN as a component of CsESPs}

rCsGRN was probed with mouse anti-His tag serum, rat anti-CsGRN serum, rat anti-CsESPs serum, and rat naïve serum as a control (Fig. 2b, Lanes 1-4). In addition, CsESPs was probed with rat anti-CsGRN serum (Lane 5) and rat naïve serum as a control (Lane 6). Rat antiCsGRN serum could specifically recognise CsESPs, and rat anti-CsESPs serum could react with $\mathrm{rCs}$ GRN (Fig. 2b, Lanes 2, 5), confirming that CsGRN was an excretorysecretory product of $C$. sinensis.

\section{Localisation of CsGRN in parasites and infection $\mathrm{Balb} / \mathrm{c}$ mouse liver}

Immunofluorescence localisation analysis showed that CsGRN was mainly localised in the tegument and testes of C. sinensis adult worm (Fig. 3).

The liver tissues from Balb/c mice infected with C.sinensis were analysed by immunohistochemistry using mouse anti-CsGRN sera (Fig. 4). Liver sections from infected and uninfected mice probed with naive serum were not shown here. Positive staining was indicated with brown. Compared with sections from normal mice, strong staining was detected in the bile duct and the liver tissue after 1 month of parasite infection. Additionally, the level of CsGRN was higher with extended time. Most importantly, brown granules were found around the cell nuclei, explaining that $C s G R N$ entered the biliary epithelium cells and hepatic cells across the cell membrane (Fig. 4). CsGRN was also seen in the bile ducts that were distant from the liver flukes, while normal mouse biliary epithelium and liver tissues were unstained (Fig. 4a, c, e, g, i, k).

\section{CsGRN overexpression in PLC-GRN and RBE-GRN cells}

The ORF of CsGRN was cloned into the pEGFP-C1 eukaryotic expression vector, and the recombinant plasmids were confirmed by sequencing (Additional file 1: Figure S1). Viable cell images of most cells showed green fluorescence and grew well after the recombinant plasmid pEGFP-C1-CsGRN transduction (Fig. 5a). To exclude the possibility that transfection plasmids could affect cell survival, the cellular viability of the transfected cells was detected by Annexin V-APC/7AAD staining with FACS analysis. Neither the pEGFP-C1 plasmids nor the pEGFPC1-CsGRN plasmids had any effect on cell viability (Fig. 5b). The qRT-PCR and Western blotting results showed the relative mRNA level and the protein expression of CsGRN were significantly raised in the PLC-GRN and RBE-GRN cells compared with that in PLC-GFP/ RBE-GFP cells (Fig. 5c, d), indicating that overexpressedCsGRN PLC/RBE cell line was successfully constructed.

\section{Cell migration and invasion triggered by CsGRN}

To determine whether CsGRN could play any role in cancer cell migration, we carried out wound-healing assays. The results showed that PLC/RBE-GRN cells could induce significant cell migration when compared with PLC/RBE-GFP cells, respectively (Fig. 6). Similarly, transwell cell migration/invasion assay results indicated that the upregulation of CsGRN in PLC and RBE cells significantly increased migration/invasive activity compared with that in the control group (Fig. 7). Therefore, CsGRN could significantly improve the migration/invasion ability of CCA and HCC in vitro.

\section{Mechanisms of CsGRN promoting CCA and HCC metastasis in vitro and in vivo}

To investigate the underlying mechanisms by which CsGRN promotes CCA and HCC cell migration and invasion, Western blotting was performed to show the expression levels of epithelial-mesenchymal transition (EMT) relevant markers. The results indicated that overexpressed-CsGRN PLC and RBE cell significantly increased vimentin, $\mathrm{N}$-cadherin and $\beta$-catenin, and 
a

c. sinensis

o. viverrini

S. japonicum

M. musculus

C. elegans

H. sapiens

F. hepatica

D. labrax

T. spiralis

S. haematobium

C. sinensis

o. viverrini

S. japonicum

M. musculus

C. elegans

H. sapiens

F. hepatica

D. labrax

T. spiralis

S. haematobium

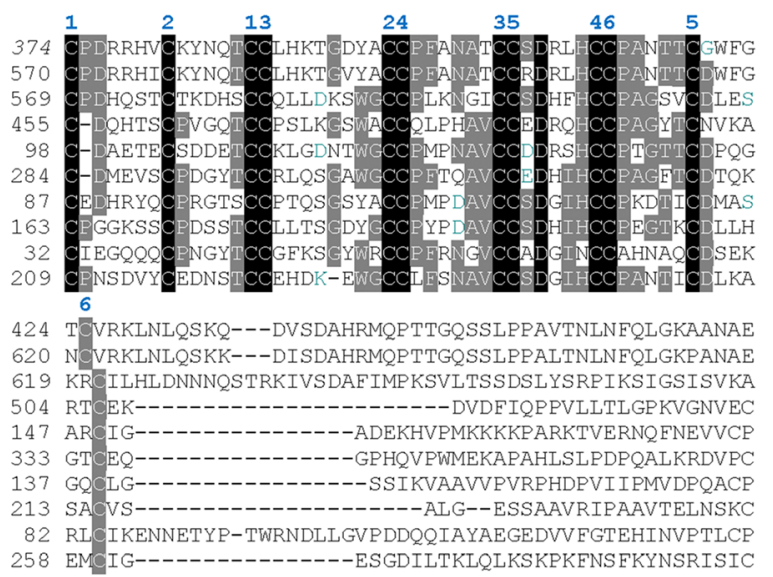

b

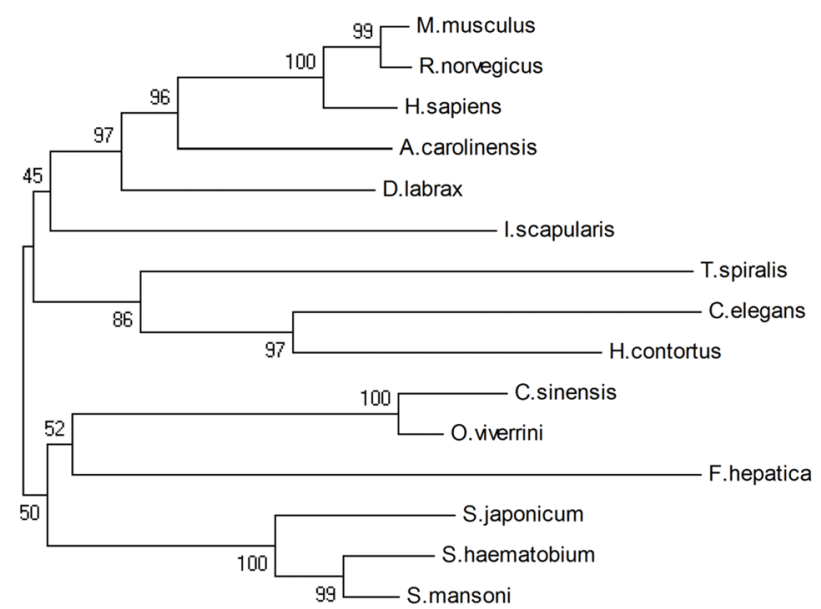

0.1

Fig. 1 a Multiple alignment of sequences with one core granulin domain from various species are performed with Vector NTI software. Identical sequences are in black and conservative sequences are in grey; theoretical disulphide bonds are numbered one to six above each cysteine residue. $\mathbf{b}$ Phylogenetic tree for the granulins from a range of phyla constructed by MEGA 6.0 using the neighbor-joining method, Poisson correction is required and bootstrap values are 1,000. The abbreviations and accession numbers of the sequences are as follows: Clonorchis sinensis (C. sinensis, GAA54205.1); Opisthorchis viverrini (O. viverrini, XP_009174632.1); Caenorhabditis elegans (C. elegans, NP_492982.1); Dicentrarchus labrax (D. labrax, CBN81737.1); Fasciola hepatica (F. hepatica, ID BN1106_s891B000441 in the WormBase ParaSite); Schistosoma japonicum (S. japonicum, CAX73857.1); Schistosoma haematobium (S. haematobium, XP_012796138.1); Homo sapiens (H. sapiens, NP_002078.1); Mus musculus (M. musculus, NP_032201.2); Rattus norvegicus (R. norvegicus, AAH72469.1); Ixodes scapularis (I. scapularis, XP_002415868.1); Trichinella spiralis (T. spiralis, XP_003371171.1); Anolis carolinensis (A. carolinensis, XP_008111426.1); Haemonchus contortus (H. contortus, CDJ86608.1), Schistosoma mansoni (S. mansoni, CCD75903.1)

decreased ZO-1 compared with the control group, indicating that CsGRN is involved in the EMT process in CCA and HCC cells (Fig. 8a, b). The relative mRNA expression of matrix metalloproteinases (MMPs) in transfected PLC and RBE cells showed that the MMP2 level was higher in PLC-GRN cells and that the MMP2 and MMP9 levels were higher in RBE-GRN cells than in PLCGFP cells or RBE-GFP cells (Fig. 8c). To further validate the effects of $C s G R N$ on promoting cancer metastasis, we conducted animal experiments. By detecting the EMTrelated indicators and relevant signalling pathway markers in $\mathrm{Balb} / \mathrm{c}$ mouse liver immunised with $\mathrm{rCs}$ GRN protein
(Fig. 8d), we found that the level of E-cadherin increased, but vimentin decreased with the immune time extended. The signal indictors p-ERK and p-AKT were used to determine the mechanisms of migration. The expression levels of p-ERK and p-AKT were dynamically changed. In detail, p-ERK reached a peak in 4 weeks after immunisation while p-AKT did in 2 weeks. The statistical data of Fig. 8a, b, d are shown in Additional file 2: Figure S2.

\section{Discussion}

Long-standing infections with $C$. sinensis eventually lead to clonorchiasis, which results in cholangiectasis, cholecystitis, 


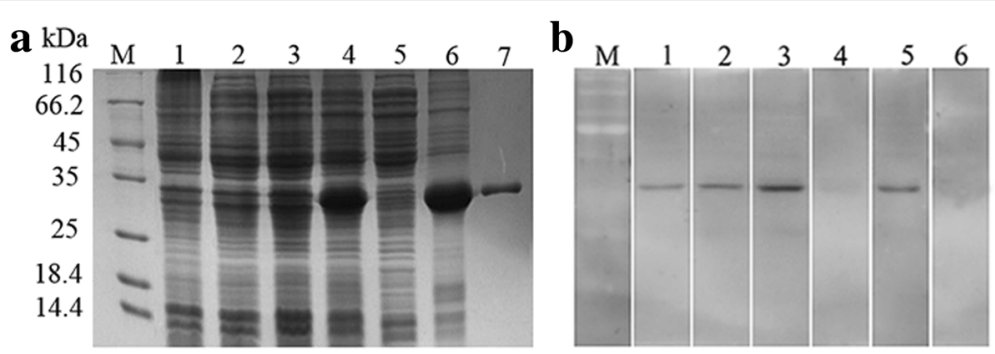

Fig. 2 Expression and identification of rCSGRN. a rCSGRN was identified by 12\% SDS-PAGE. Protein molecular weight markers (Lane M), lysate of E. coli containing pET-28a (+) without induction (Lane 1) and with induction by IPTG (Lane 2), lysate of E. coli containing pET-28a (+)-CSGRN without induction (Lane 3) and with induction by IPTG (Lane 4), supernatant (Lane 5) and precipitate (Lane 6) of the lysate of E. coli containing the recombinant plasmid after induction, the purified recombinant CSGRN protein (Lane 7). $\mathbf{b}$ rCSGRN protein was identified as a component of CSESPS. rCSGRN protein was probed by mouse anti-His-tag serum, rat anti-CsGRN serum, rat anti-CsESPs serum and naïve serum (Lanes 1-4, respectively). In addition, CsESPs were probed with rat anti-CsGRN serum and naïve serum (Lanes 5, 6). Protein molecular weight markers (Lane M)

cholelithiasis, hepatic fibrosis, and even CCA and HCC [22]. Additionally, researchers have proposed that mechanical damage caused by the activities of the parasites and CsESPs excreted and secreted from the liver fluke is the primary pathogenic mechanism [23]. In our previous studies [24], CsGRN, one of an ingredient of CsESPs, was identified and the prokaryotic expression of the recombinant $C s G R N$ protein was carried out.

Sequence analysis showed that CsGRN was a granulin member that has $28 \%$ overall identity with human PGRN. It shared the characteristic granulin motif that was reported to be a potent proliferative agent. Additionally,

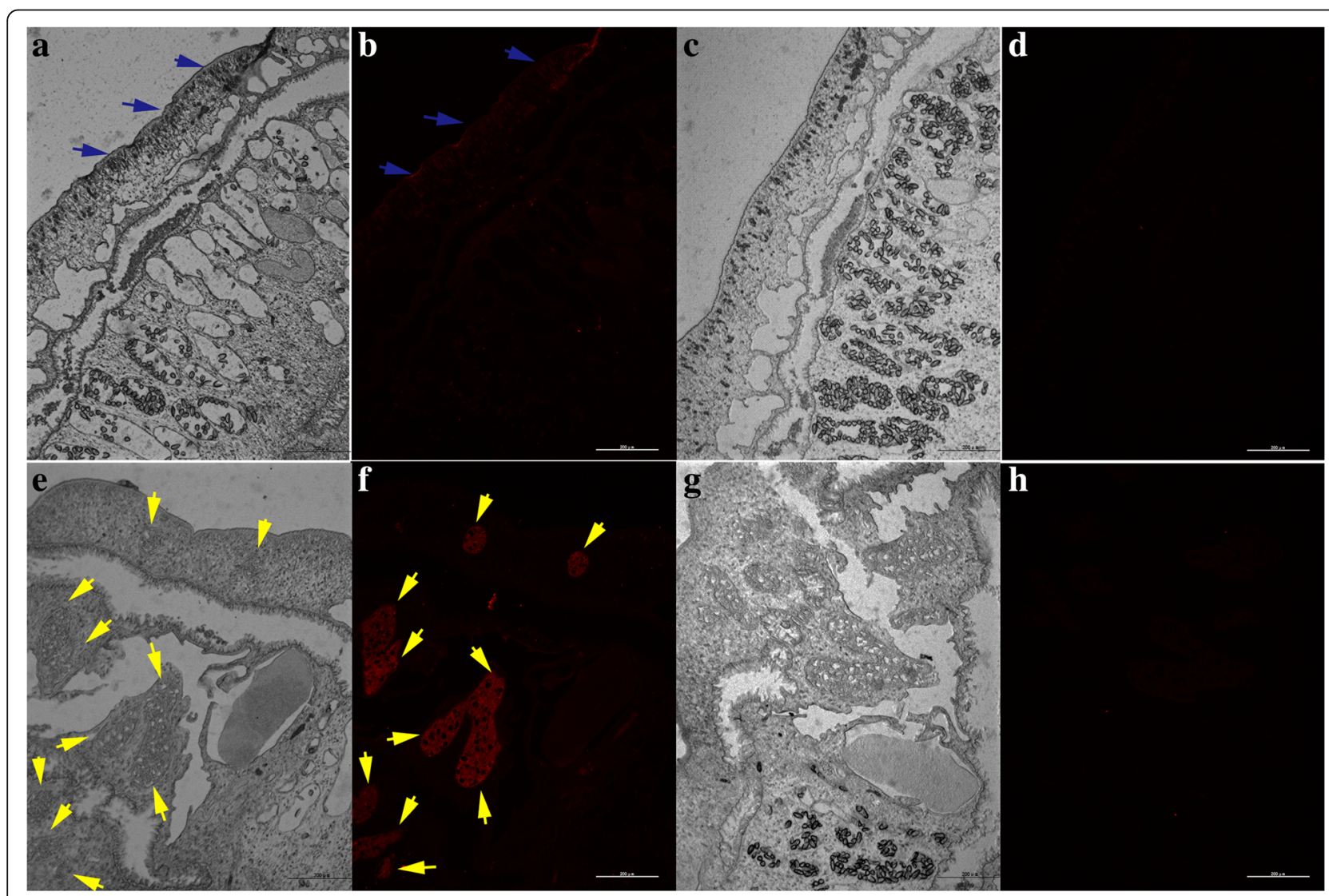

Fig. $\mathbf{3}$ Immunolocalization of CSGRN in C. sinensis adult worm. Sections of C. sinensis adult worm were probed with rat anti-CSGRN serum (a, $\mathbf{b}$, e, $\mathbf{f})$ or normal rat sera $(\mathbf{c}, \mathbf{d}, \mathbf{g}, \mathbf{h})$ followed by my3-conjugated anti-rat lgG. Sections were imaged under fluorescence light $(\mathbf{b}, \mathbf{d}, \mathbf{f}, \mathbf{h})$, or white light $(\mathbf{a}, \mathbf{c}, \mathbf{e}, \mathbf{g})$. CsGRN was distributed in the tegument and testes of the adult worm $(\mathbf{b}, \mathbf{f})$. Blue arrow: tegument; Yellow arrow: testes. The images were magnified at 100× (scale-bar: $200 \mu \mathrm{m}$ ) for adult worm 


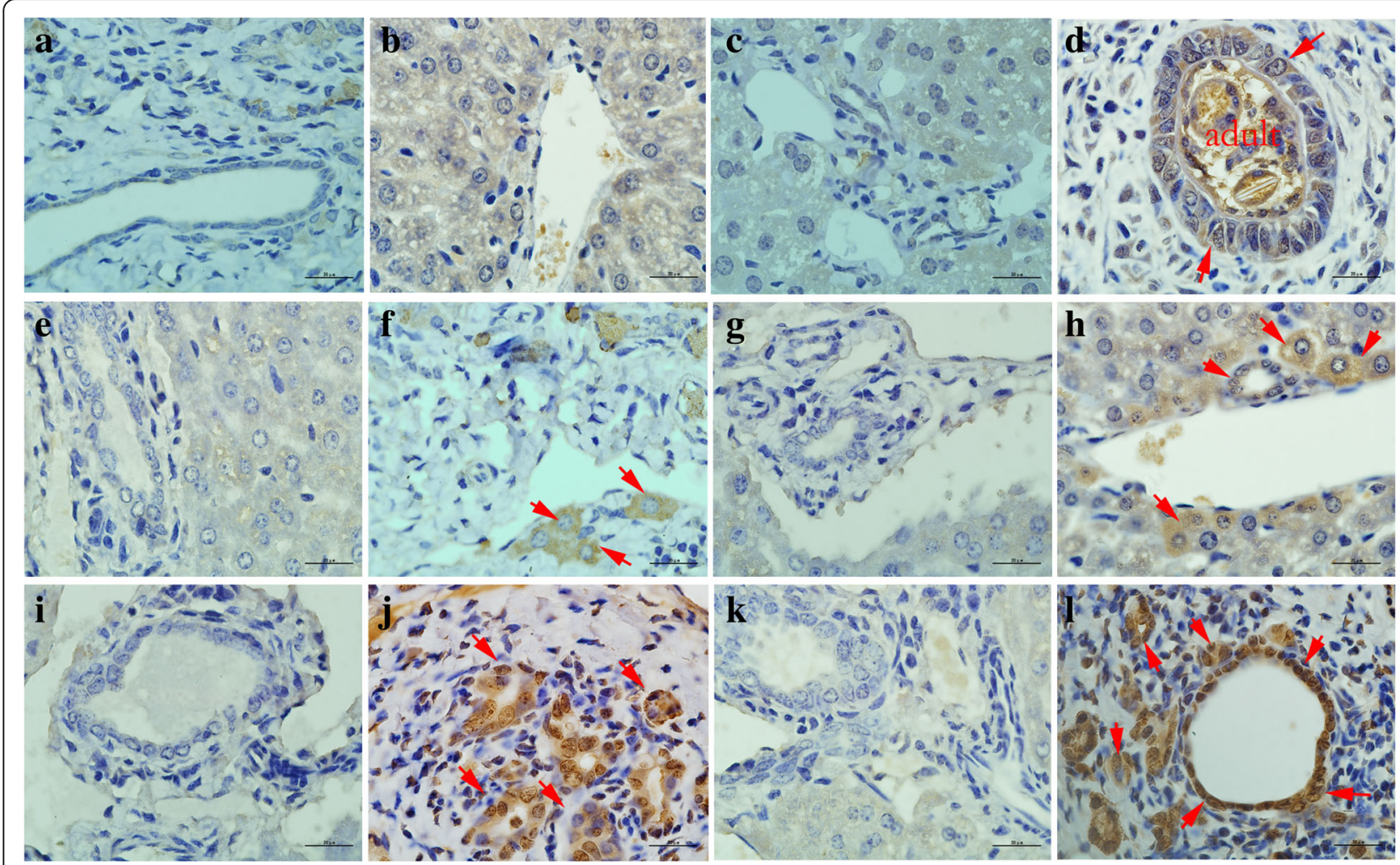

Fig. 4 Immunohistochemical localisation of CSGRN in infected Balb/c mouse livers. Sections of Balb/c mouse livers infected with C. sinensis treated with mouse anti-CsGRN serum (b, d, $\mathbf{f}, \mathbf{h}, \mathbf{j}$ and $\mathbf{I}$ ) or mouse naïve serum (a, c, e, g, i and $\mathbf{k}$ ). During early infection (1 month), CsGRN was observed mainly surrounding the bile ducts where parasites reside, and brown granules were found around cell nuclei, which explained how CsGRN entered the biliary epithelium cells across the cell membrane (d). From 2 months post-infection, CsGRN was also seen within the hepatocytes (f, h, j and I). With prolonged infection, more CsGRN gathered in bile duct epithelial cells and hepatocytes. Positive hepatocytes and biliary epithelium cells were even found in the smaller bile duct where adult worm could not reach. $\mathbf{a}, \mathbf{b}$ normal, $\mathbf{c}, \mathbf{d} 1$ month, e, $\mathbf{f} 2$ months, $\mathbf{g}$, $\mathbf{h}$ 3 months, $\mathbf{i}, \mathbf{j} 4$ months and (k, I) 6 months post-infection. Arrows indicate positive cells. The images were magnified at 1,000x (scale-bar: 20 um)

CsGRN may promote carcinoma progression by prompting angiogenesis, insensitivity to apoptosis, tumour invasion and anchorage independence, all of which support tumour expansion in the unfavourable interstitial environment $[25,26]$. Although the deduced protein was predicted without signal peptides, the localisation of CsGRN in the tegument and testes of $C$. sinensis adult worm indicated that the protein could be secreted through other pathways such as that used by F. hepatica [14].

The protein was also found in the hepatocytes and cholangiocytes of infected Balb/c mice, possibly implying CsGRN could be internalised by various mammalian cells via an endocytotic mechanism [27-29]. Moreover, the surprising finding provided a clue about the protein pertaining to its function on interaction with host cells as an ingredient of CsESPs. According to some reports, granulin plays a central role in the carcinogenesis of a range of malignancies [9]. For instance, PGRN is highly expressed in ovarian tumours [30], breast cancer [31], glioblastomas [32], and gastric cancer [33]. Moreover, as an important mediator of tumorigenesis and wound repair, it is associated with aggressive and invasive tumour phenotypes by stimulating invasion, protecting against anoikis, and supporting tumour expansion in the unfavourable interstitial environment $[34,35]$. Based on these aspects, the overexpressed-CsGRN stable cell lines named as PLC-GRN and RBE-GRN cells were successfully constructed, and the wound-healing assay and transwell assay were performed to observe the ability of the migration and invasion of CCA and HCC in vitro. The results displayed that they could stimulate cancer cell migration, which was similar to the action exerted by other recombinant proteins [36, 37]. Compared with cells co-cultured with protein, PLC-GRN and RBE-GRN cells were more approximate to the real situation that the internalised protein of this parasite might interfere with signalling and displayed the carcinogenic nature of biliary epithelial cells and hepatocytes. Likewise, Helicobacter pylori CagA protein targets PAR1/MARK kinase after delivery into gastric epithelial cells and disrupts cell polarity, resulting in disorganisation of the gastric epithelial architecture, inflammation and carcinogenesis 

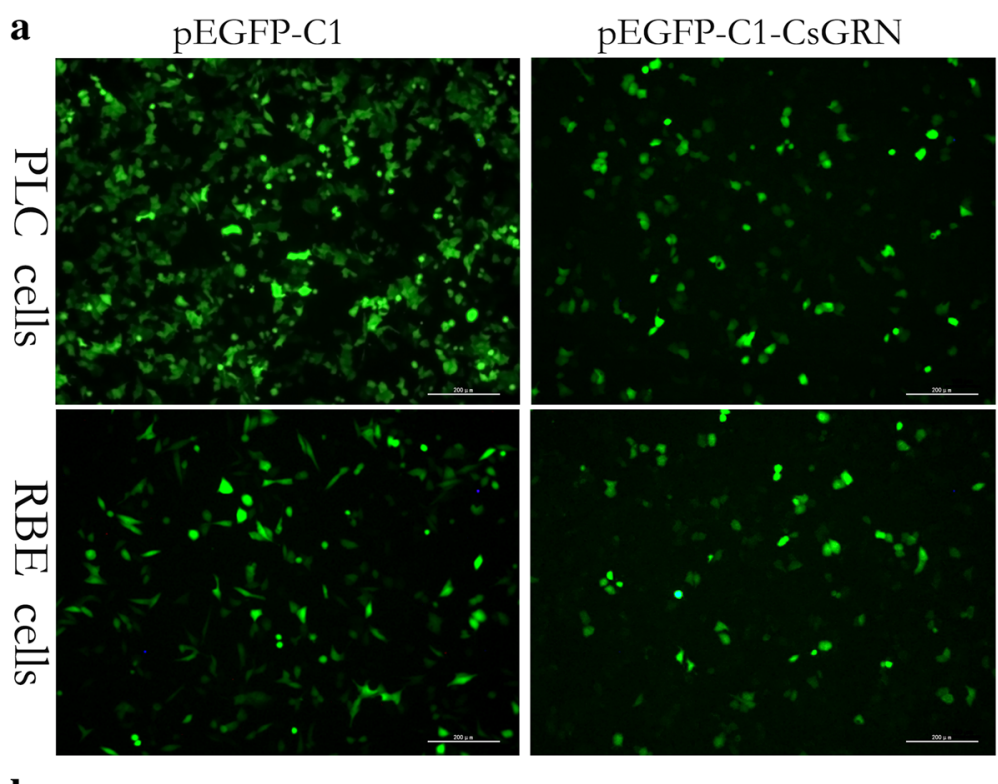

b
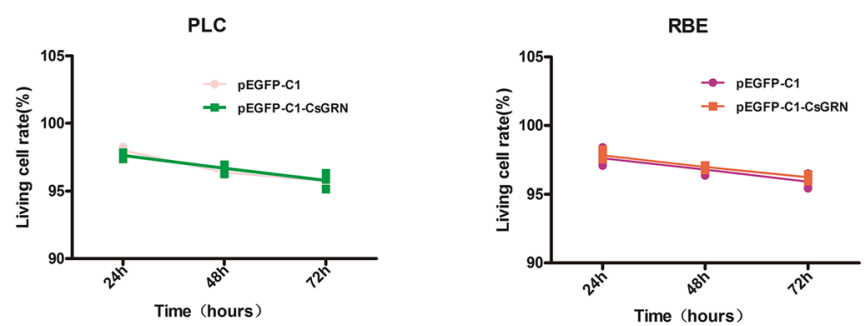

C
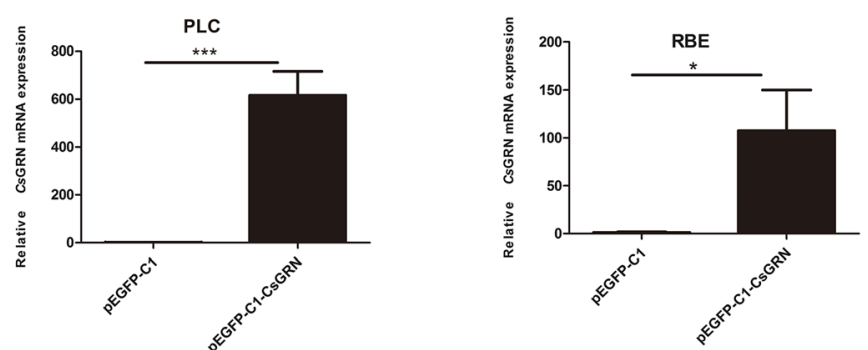

d
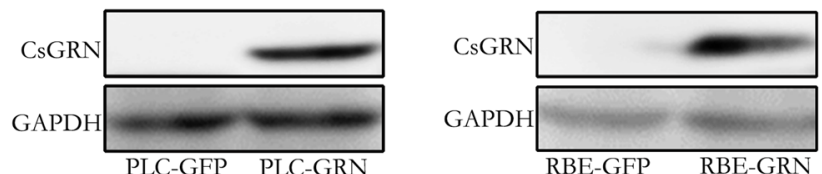

Fig. 5 Successful construction of stable CsGRN-overexpressed hepatoma cells and cholangiocarcinoma cells. a Green fluorescence was observed under an inverted microscope and was emitted by PLC and RBE cells transformed with pGFP-C1 and pGFP-C1-CsGRN, respectively. The images were magnified at 100x (scale-bar: $200 \mu \mathrm{m}$ ).b Cellular viability of transfected PLC and RBE cells ( $24 \mathrm{~h}, 48 \mathrm{~h}$ and $72 \mathrm{~h}$ after transfection) was detected by Annexin V-APC/7AAD double staining with FACS analysis. c qRT-PCR was used to detect the expression of CSGRN mRNA between the experimental group and control group. $\mathbf{d}$ Western blot analysis was used to determine the CSGRN protein expression between the experimental group and control group. ${ }^{*} P<0.05,{ }^{* *} P<0.001$ compared with a matched group

[38]. Furthermore, similar to lipoprotein, internalisation induced by activating the fuel-sensing enzyme adenosine monophosphate-activated protein kinase (AMPK) is crucial to maintaining glioblastoma cell growth [39].

However, the mechanisms by which CsGRN enhances tumour metastasis are unclear. And we hypothesised that they could be pertaining to EMT process, which endows cancer cells with a more aggressive phenotype, finally leading to tumour progression, including invasion and metastasis $[40,41]$. Various biomarkers have been screened to demonstrate the EMT process, including the upregulation of mesenchymal markers such as $\mathrm{N}$-cadherin, $\beta$-catenin 


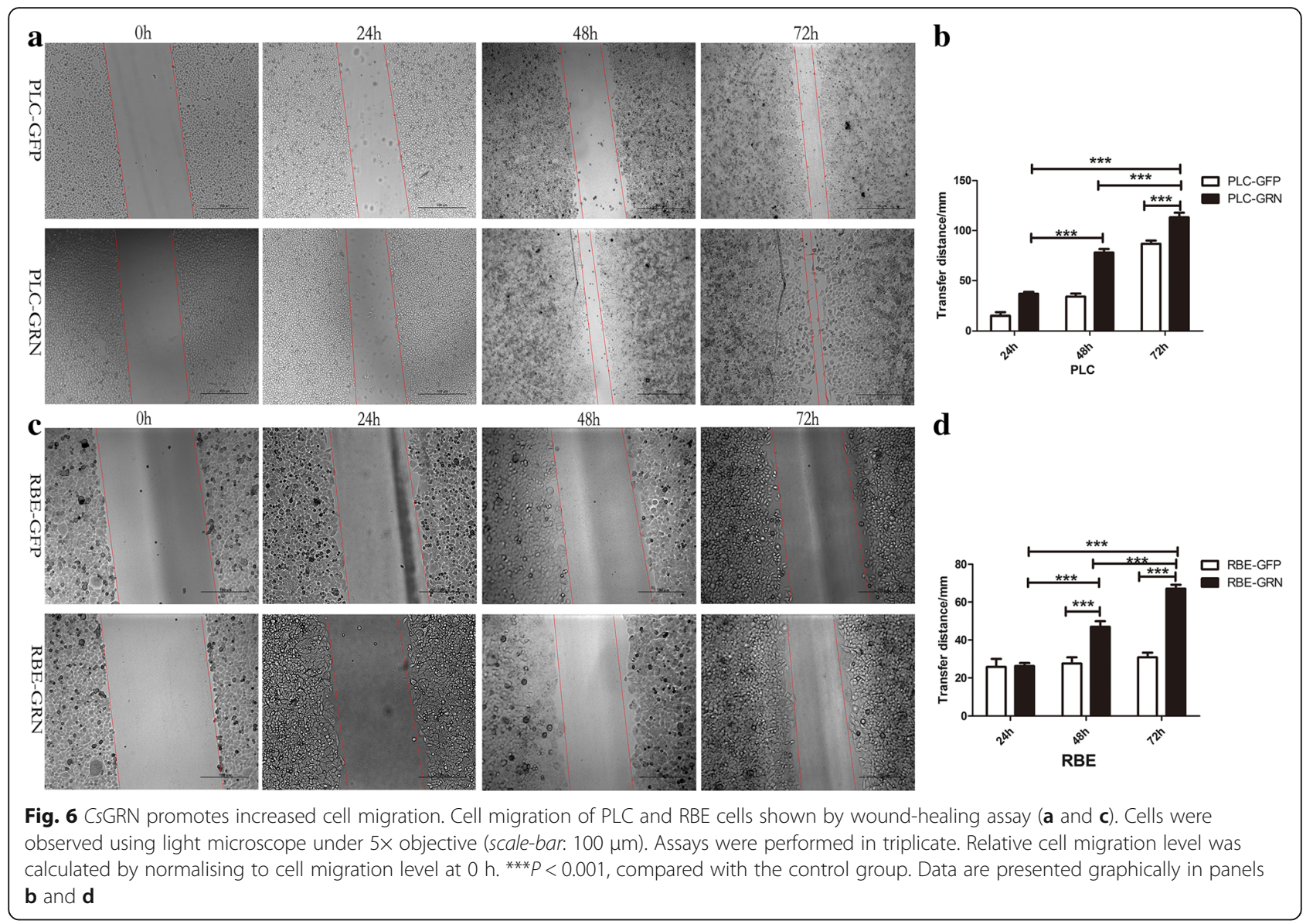

and vimentin, as well as the loss of epithelial markers such as E-cadherin and ZO-1 [42].

Cadherins are transmembrane glycoproteins of cellular junctions that mediate calcium-dependent cell-cell adhesion and alterations in cadherin function have been implicated in tumorigenesis [43]. Although the loss of E-cadherin has been seen as a hallmark of EMT, recent evidence have indicated that a gain of expression of $\mathrm{N}$ cadherin but without E-cadherin change in tumour cells contributes directly to an increased invasive potential and is independently associated with an early stage of metastasis $[44,45]$. The catenins, serving to link the cadherin to the cytoskeleton, can regulate alterations in cadherin function [46]. Disruption of the connection between the cadherins and the cytoskeleton by mutations in $\beta$-catenin inactivates the adhesive function of E-cadherin in tumour cells and results in a non-adhesive phenotype. Thus, the increased expression of $\beta$-catenin is intimately related to the decline of E-cadherin [47]. Our experiments confirm these findings. Similarly, in PLC-GRN/RBE-GRN cells, the up-modulation of $\mathrm{N}$-cadherin, $\beta$-catenin and vimentin, as well as the downregulation of ZO-1while the decrease of E-cadherin were not detected in vitro. Therefore, we can also draw the conclusion that CsGRN induced the mesenchymal characteristics of PLC and RBE cells in responsible for cell invasion and metastasis. In contrast, in the liver of $B a l b / c$ mice immunised with $\mathrm{rCs} G \mathrm{RN}$ protein, the upregulation of E-cadherin and downregulation of vimentin indicated the block of EMT progress. In other words, anti-CsGRN, a specific antibody produced by immunisation with $\mathrm{rCs} G \mathrm{RN}$ protein, probably prevents the tumour cells from invasion and metastasis.

As many studies have reported, MMPs play a predominant role in the process of tumour cell intravasation, and the dynamic interplay between $\mathrm{N}$-cadherin and epidermal growth factor receptor (EGFR) leads to MMPs gene transcription [48]. As shown in our data, the expression of $\mathrm{N}$-cadherin and MMPs was increased in PLC-GRN/RBEGRN cells; therefore, we speculated that the level of MMPs was increased under the action of the $\mathrm{N}$-cadherin and EGFR through stimulation by CsGRN, eventually leading to the degradation of basement membranes and metastasis.

Numerous studies have shown that $\beta$-catenin, a key downstream effector in the Wnt signalling pathway, is considered to be a cell-cell adhesion protein, and most likely promotes tumour progression once activated [49]. We investigated the overexpression of $\beta$-catenin in vitro but without a change in vivo depending on our study, 


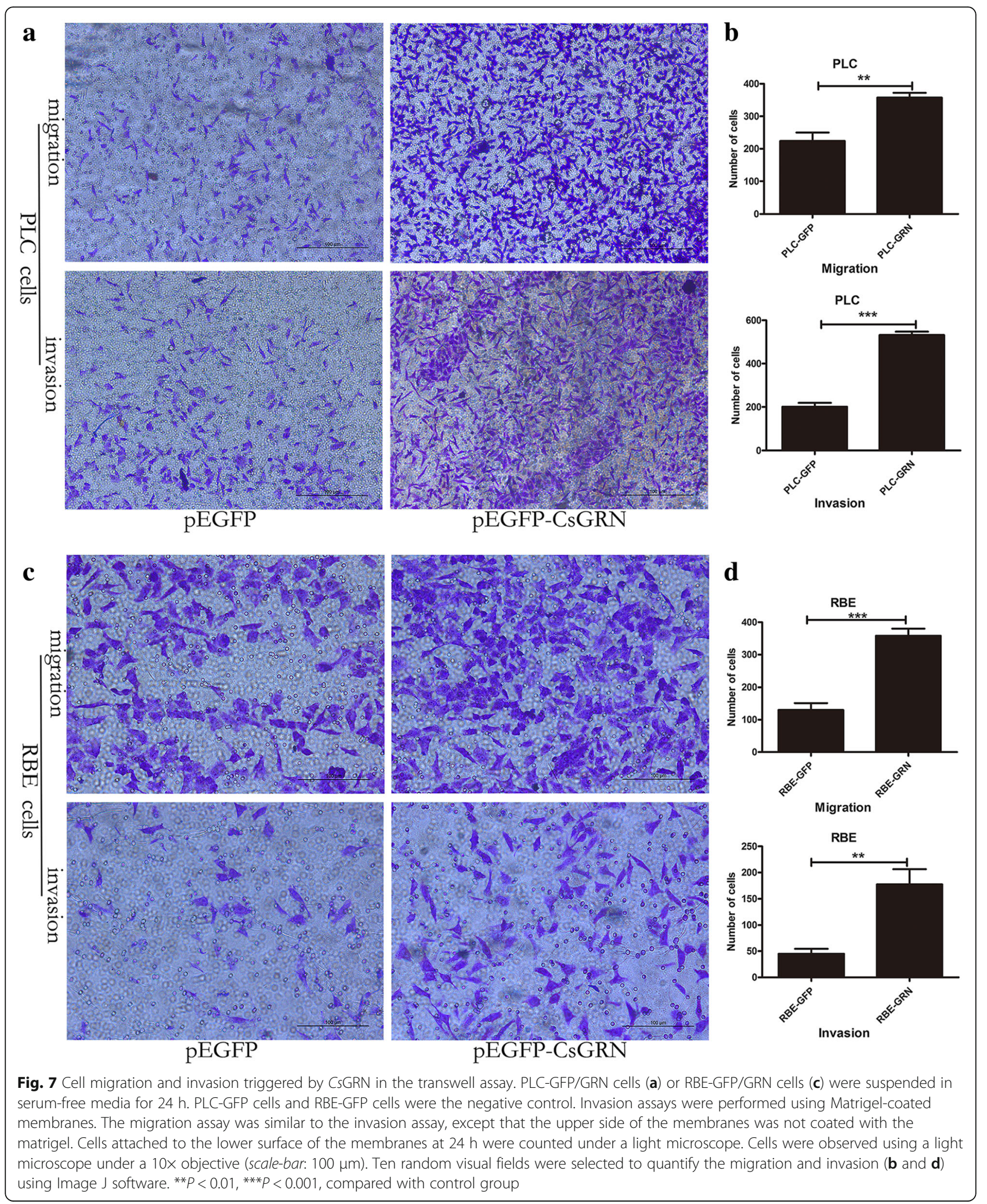

which prompted the speculation that $\beta$-catenin only acted as a biomarker of EMT but didn't activate the $\beta$ catenin/wnt pathway.
To further study the potential downstream effectors modulated by CsGRN promotion progression of HCC and CCA, we also tested the signalling molecules AKT 
a

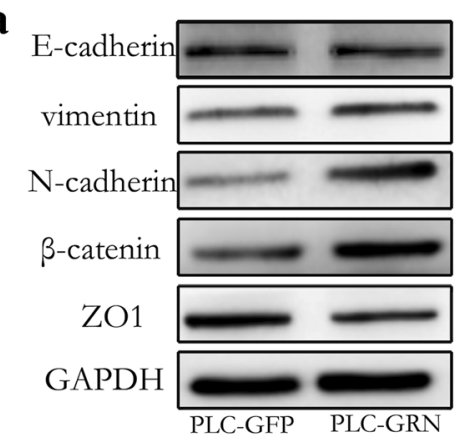

c

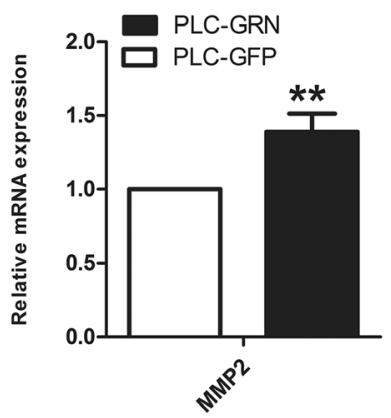

b
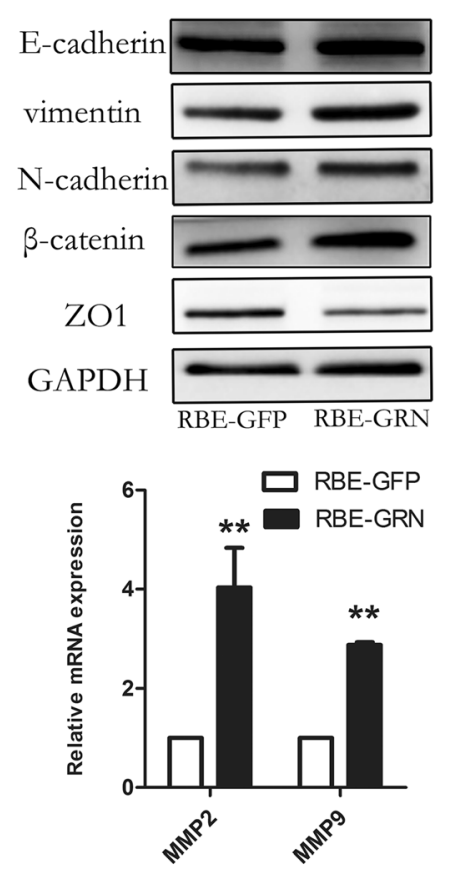

d
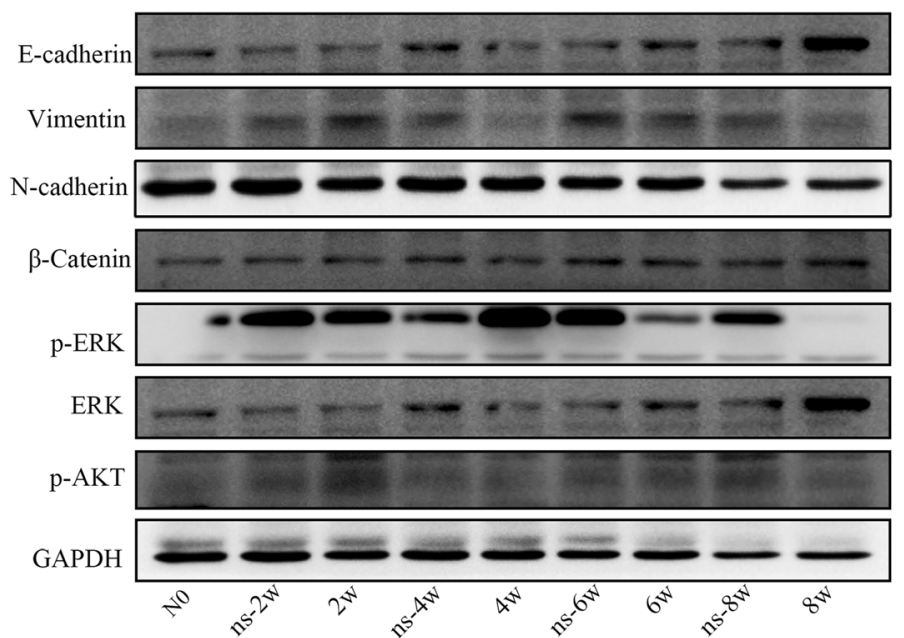

Fig. 8 CSGRN promotes mesenchymal characteristics. a, b Western blotting was used to detecting the expression of EMT-relevant markers in transfected PLC and RBE cells. PLC-GRN/RBE-GRN cells showed the upregulation of vimentin, N-cadherin and $\beta$-catenin, and downregulation of ZO-1; however, the expression level of E-cadherin had not changed. c Relative mRNA expression of MMPs in transfected PLC and RBE cells. MMP2 expression was higher in PLC-GRN cells, and MMP2 and MMP9 were higher in RBE-GRN cells than in PLC-GFP cells or RBE-GFP cells. ${ }^{* *} P<0.01$, compared with control group. $\mathbf{d}$ The expression of EMT-relevant markers and relevant signalling pathways indicators was detected in the liver from Balb/c mice injected with rCsGRN protein. The level of E-cadherin increased, and the level of vimentin decreased in rCsGRN protein-treated mice with an extended immune time extended (no change in $\mathrm{N}$-cadherin). The expression levels of $\mathrm{p}$-ERK and $\mathrm{p}$-AKT were dynamically changed. p-ERK reached a peak in 4 weeks after immunisation, while p-AKT did at 2 weeks

and ERK using Western blotting. Our results showed that the expression of p-ERK and p-AKT were dynamically changed when p-ERK reached a peak in 4 weeks after immunisation while $\mathrm{p}$-AKT did at 2 weeks. These data implied that the PI3K/AKT and ERK pathways participated in the promotion of EMT induced by CsGRN, and they might not be activated at the same time.
As we know, the disease is often caused by complicated factors. This study observed that CsGRN, as a CsESP like CsCBs and Csseverin, has also been shown to promote cell migration and invasion. In consideration of the complex constitution of $C s E S P s$, including proteases, antioxidant enzymes and metabolic enzymes [24, 50], interactions among these components are required to 
determine the authentic factors for pathogenesis in the future. We will explore the actions of granulin members with one or more typical domains in C. sinensis to clarify the difference between these molecules in our further studies.

Overall, we identified CsGRN was a growth factor of C. sinensis and a vital constituent of CsESPs. To date, we have observed the potential promotion cell migration and invasion of CsGRN. We also showed that the EMT process can be triggered by $C s \mathrm{GRN}$ and involved in HCC and CCA metastasis via the activation of the PI3K/ AKT and ERK pathways.

\section{Conclusions}

In summary, we identified CsGRN as belonging to the granulin family through bioinformatics and phylogenetic analyses. We also expressed and purified soluble rCsGRN in E.coli and discovered it was an important component of CsESPs. In adult worm, CsGRN is mainly located in the tegument and testes, which might be involved in parasite growth and development or even in the pathopoiesis of the parasite. CsGRN could be detected at a high expression level in clonorchiasis-induced $\mathrm{Balb} / \mathrm{c}$ mouse liver tissues and was even observed in hepatocytes and cholangiocytes. In addition, the secretory eukaryotic expression vector pEGFP-C1-CsGRN was generated and employed to intervene in the expression and secretion of CsGRN in PLC and RBE cells. An enhancement of cell migration and invasion was observed, and the current results suggested that CsGRN is likely to promote cell migration and invasion by inducing liver EMT via the ERK and PI3K/AKT signalling pathways. The present study supports the involvement of CsGRN in the pathogenesis of CCA and HCC.

\section{Additional files}

Additional file 1: Figure S1. Successful construction of the eukaryotic expression plasmid pEGFP-C1-CsGRN. a Restriction enzyme identification of the recombinant plasmid pEGFP-C1-CsGRN. DNA ladder 5000 (Lane M), double enzyme digestion of pEGFP-C1-CSGRN (Lane 1), recombinant plasmid pEGFP-C1-CSGRN (Lane 2), empty vector pEGFP-C1 (Lane 3). b Sequencing data from recombinant plasmid pEGFP-C1-CSGRN and CSGRN gene were completely matched. (TIF $206 \mathrm{~kb}$ )

Additional file 2: Figure S2. Densitometric analysis of genes from Fig. $8 \mathbf{a}, \mathbf{b}, \mathbf{d}$. Densitometric results were analysed with Image J software. Statistical comparisons between more than two experimental groups were made with one-way ANOVA tests followed by Tukey's multiple comparisons test. Results are reported as the mean \pm standard error of the mean (SEM), and $P$ was set to 0.05 . For all analyses, Prism 5.0 software (Graph Pad Software, San Diego, USA) was used. a ${ }^{*} P<0.05$, ${ }^{* *} P<0.01$, compared with the control group. $b{ }^{*} P<0.05$, ${ }^{* *} P<0.01$ and ${ }^{* * *} P<0.001$, indicate difference from experimental treatment. ${ }^{\# \#} P<0.01$ and ${ }^{\# \#} P<0.001$, compared with the matched pair. (TIF $910 \mathrm{~kb}$ )

\section{Abbreviations}

AMPK: Adenosine monophosphate-activated protein kinase; CCA: Cholangiocarcinoma; CsGRN: Clonorchis sinensis granulin; EGFR: Epidermal growth factor receptor; EMT: Epithelial to mesenchymal transition; ERK: Extracellular signal-regulated kinase; ESPs: Excretory/secretory products; HCC: Hepatocellular carcinoma; MMP2 and MMP9: Matrix metalloproteinase 2 and 9; MMPs: Matrix metalloproteinases;

PGRN: Progranulin; PI3K: Phosphatidyl inositol 3-kinase

\section{Acknowledgements}

We appreciate MD. Guping Mao, PhD. Zhiyu Huang, Prof. Weiming Liao and Prof. Yan Kang (Department of Orthopaedi in First Affiliated Hospital of Sun Yat-sen University) for providing help to us.

\section{Funding}

This work was supported by the National Key Research and Development Program of China (Nos. 2016YFC1202003, 2016YFC1202005), National Natural Science Foundation of China (No.81641094) and Guangdong Natural Science Foundation (No. S2012010008504) to XL, the National Key Basic Research Program of China (No.2010CB530000), the Guangdong Natural Science Fund (No. S2012010008504), and National Natural Science Foundation of China (No. 81171602) to XY. This work was supported in part by National Natural Science Foundation of China (No. 81101270) to YH.

\section{Availability of data and materials}

The datasets generated and analysed during the current study are available in GenBank under accession number KY855531.

\section{Authors' contributions}

CW, HL, MShang, YT, ZL, XiaoT, YH, XY and XL conceived and designed the experiments; $C W, H L, M S h a n g, Y T, Y W, Y L$ and $L Z$ performed the

experiments; CW, HL, YT, MShi, XinT, TC and XL analyzed the data; CW, HL, $Y H, X Y$ and $X L$ wrote the manuscript. All authors read and approved the final manuscript.

\section{Competing interests}

The authors declare that they have no competing interests.

\section{Consent for publication}

Not applicable.

\section{Ethics approval and consent to participate}

Balb/c mice and Sprague-Dawley (SD) rats were purchased from the Animal Center of Sun Yat-sen University (Guangzhou, China) and raised strictly in accordance with National Institutes of Health on animal care and ethical guidelines. The experimental procedures were approved by the animal care and use committee of Sun Yat-sen University (Permit Numbers: SCXK (Guangdong) 2016-105XS).

\section{Publisher's Note}

Springer Nature remains neutral with regard to jurisdictional claims in published maps and institutional affiliations.

\section{Author details}

${ }^{1}$ Department of Parasitology, Zhongshan School of Medicine, Sun Yat-sen University, Guangzhou 510080, People's Republic of China. ${ }^{2}$ Key Laboratory for Tropical Diseases Control of Ministry of Education, Sun Yat-sen University, Guangzhou 510080, People's Republic of China. ${ }^{3}$ Provincial Engineering Technology Research Center for Biological Vector Control, Guangzhou 510080, People's Republic of China. ${ }^{4}$ Research Institute of Infectious Diseases, Guangzhou Eighth People's Hospital, Guangzhou Medical University, Guangzhou 510060, People's Republic of China.

Received: 3 December 2016 Accepted: 8 May 2017

Published online: 25 May 2017

References

1. Qian MB, Utzinger J, Keiser J, Zhou XN. Clonorchiasis. Lancet Infect Dis. 2016;10020:800-10.

2. Wu Y, Li Y, Shang M, Jian Y, Wang C, Bardeesi ASA, et al. Secreted phospholipase A2 of Clonorchis sinensis activates hepatic stellate cells through a pathway involving JNK signalling. Parasit Vectors. 2017;10:147.

3. Huang Y, Chen W, Wang X, Liu H, Guo L, Luo F, et al. The carcinogenic liver fluke, Clonorchis sinensis: new assembly, reannotation and analysis of the genome and characterization of tissue transcriptomes. PLoS One. 2013;8:e54732. 
4. Tan SK, Qiu XQ, Yu HP, Zeng XY, Zhao YN, Hu L. Evaluation of the risk of clonorchiasis inducing primary hepatocellular carcinoma. Zhonghua Gan Zang Bing Za Zhi. 2008;16(2):114-6.

5. Xiao X, Liang $X$, Zhu S. Studies on primary hepatoma cases and nonhepatoma cases infested by clonorchiasis. Journal of Jinan University. 1985:4(1):16-8.

6. Kim Yl. Liver carcinoma and liver fluke infection. Arzneimittelforsch. 1984;34(9b):1121-6.

7. Wang X, Chen W, Huang Y, Sun J, Men J, Liu H, et al. The draft genome of the carcinogenic human liver fluke Clonorchis sinensis. Genome Biol. 2011;12(10):R107.

8. Young ND, Campbell BE, Hall RS, Jex AR, Cantacessi C, Laha T, et al. Unlocking the transcriptomes of two carcinogenic parasites, Clonorchis sinensis and Opisthorchis viverrini. PLoS Negl Trop Dis. 2010;4(6), e719.

9. Bateman A, Bennett HP. The granulin gene family: from cancer to dementia. Bioessays. 2009:31:1245-54

10. Ong CH, Bateman A. Progranulin (Granulin-epithelin precursor, PC-cell derived growth factor, Acrogranin) in proliferation and tumorigenesis. Histol Histopathol. 2003;1:1275-88.

11. Yip CW, Cheung PF, Leung IC, Wong NC, Cheng CK, Fan ST, et al. Granulinepithelin precursor interacts with heparan sulfate on liver cancer cells. Carcinogenesis. 2014;35:2485-94.

12. Smout MJ, Laha T, Mulvenna J, Sripa B, Suttiprapa S, Jones A, et al. A granulin-like growth factor secreted by the carcinogenic liver fluke, Opisthorchis viverrini, promotes proliferation of host cells. PLoS Pathog 2009;5(10):e1000611.

13. Papatpremsiri A, Smout MJ, Loukas A, Brindley PJ, Sripa B, Laha T. Suppression of Ov-grn-1 encoding granulin of Opisthorchis viverrini inhibits proliferation of biliary epithelial cells. Exp Parasitol. 2015;148:17-23.

14. Machicado C, Marcos LA, Zimic M. Hypothetical granulin-like molecule from Fasciola hepatica identified by bioinformati analysis. Springerplus. 2016;5(1):773.

15. Zheng S, Zhu Y, Zhao Z, Wu Z, Okanurak K, Lv Z. Liver fluke infection and cholangiocarcinoma: a review. Parasitol Res. 2016;116:11-19.

16. Chen X, Li S, He L, Wang X, Liang P, Chen W, et al. Molecular characterization of severin from Clonorchis sinensis excretory/secretory products and its potential anti-apoptotic role in hepatocarcinoma PLC cells. PLoS Negl Trop Dis. 2013;7(12):e2606.

17. Chen W, Ning D, Wang X, Chen T, Lv X, Sun J, et al. Identification and characterization of Clonorchis sinensis cathepsin B proteases in the pathogenesis of clonorchiasis. Parasit Vectors. 2015:8:647.

18. Hu F, Yu X, Ma C, Zhou H, Zhou Z, Li Y, et al. Clonorchis sinensis: expression, characterization, immunolocalization and serological reactivity of one excretory/ secretory antigen-LPAP homologue. Exp Parasitol. 2007;117(2):157-64.

19. Tamura K, Stecher G, Peterson D, Filipski A, Kumar S. MEGA6: Molecular Evolutionary Genetics Analysis version 6.0. Mol Biol Evol. 2013;30(12):2725-9.

20. Jonkman JE, Cathcart JA, Xu F, Bartolini ME, Amon JE, Stevens KM, et al. An introduction to the wound healing assay using live-cell microscopy. Cell Adh Migr. 2014;8(5):440-51

21. Marshall J. Transwell ( ${ }^{\circledR}$ ) invasion assays. Methods Mol Biol. 2011;769:97-110.

22. Qian MB, Utzinger J, Keiser J, Zhou XN. Clonorchiasis. Lancet. 2016;387(10020):800-10.

23. Pak JH, Kim DW, Moon JH, Nam JH, Kim JH. Differential gene expression profiling in human cholangiocarcinoma cells treated with Clonorchis sinensis excretory-secretory products. Parasitol Res. 2009;5:1035-46.

24. Wang $X$, Hu F, Hu X, Chen W, Huang Y. Proteomic identification of potential Clonorchis sinensis excretory/secretory products capable of binding and activating human hepatic stellate cells. Parasitol Res. 2014;113:3063-71.

25. Yung MK, Lo KW, Yip CW, Chung GT, Tong CY. Copy number gain of granulin-epithelin precursor (GEP) at chromosome 17q21 associates with overexpression in human liver cancer. BMC Cancer. 2015;2:264.

26. Marsh T, Wong I, Sceneay J, Barakat A, Qin Y, Sjodin A, et al. Hematopoietic age at onset of triple-negative breast cancer dictates disease aggressiveness and progression. Cancer Res. 2016;10:2932-43.

27. Kumari S, Mg S, Mayor S. Endocytosis unplugged: multiple ways to enter the cell. Cell Res. 2010;20(3):256-75.

28. Matchimakul P, Rinaldi G, Suttiprapa S, Mann VH, Popratiloff A, Laha T, et al. Apoptosis of cholangiocytes modulated by thioredoxin of carcinogenic liver fluke. Int J Biochem Cell Biol. 2015:65:72-80.

29. Suttiprapa S, Loukas A, Laha T, Wongkham S, Kaewkes S, Gaze S, et al. Characterization of the antioxidant enzyme, thioredoxin peroxidase, from the carcinogenic human liver fluke, Opisthorchis viverrini. Mol Biochem Parasit. 2008;160(2):116-22

30. Cuevas-Antonio R, Cancino C, Arechavaleta-Velasco F, Andrade A, Barron L, Estrada I, et al. Expression of progranulin (Acrogranin/PCDGF/GranulinEpithelin Precursor) in benign and malignant ovarian tumors and activation of MAPK signaling in ovarian cancer cell line. Cancer Invest. 2010;5:452-8.

31. Kim WE, Yue B, Serrero G. Signaling pathway of GP88 (Progranulin) in breast cancer cells: upregulation and phosphorylation of c-myc by GP88/ Progranulin in Her2-overexpressing breast cancer cells. Breast Cancer (Auckl). 2015;9 Suppl 2:71-7.

32. Wang M, Li G, Yin J, Lin T, Zhang J. Progranulin overexpression predicts overall survival in patients with glioblastoma. Med Oncol. 2012;29(4):2423-31.

33. Wang H, Sun Y, Liu S, Yu H, Li W, Zeng J, et al. Upregulation of progranulin by Helicobacter pylori in human gastric epithelial cells via p38MAPK and MEK1/2 signaling pathway: role in epithelial cell proliferation and migration. FEMS Immunol Med Mic. 2011;63(1):82-92.

34. Zhao $M$, He M, Huang $X$, Wang $Q$, Shi Y. Functional characterization and molecular mechanism exploration of three granulin epithelin precursor splice variants in biomineralization of the pearl oyster Pinctada fucata. Mol Genet Genomi. 2016:1:399-409.

35. Tangkeangsirisin W, Seeerro G. PC cell-derived growth factor (PCDGF/GP88, progranulin) stimulates migration, invasiveness and VEGF expression in breast cancer cells. Carcinogenesis. 2004;9:1587-92.

36. Smout MJ, Sotillo J, Laha T, Papatpremsiri A, Rinaldi G, Pimenta RN, et al. Carcinogenic parasite secretes growth factor that accelerates wound healing and potentially promotes neoplasia. PLoS Pathog. 2015;11(10), e1005209.

37. Botelho MC, Alves H, Richter J. Wound healing and cancer progression in Opisthorchis viverrini associated cholangiocarcinoma. Parasitol Res. 2016;115(7):2913-4.

38. Saadat I, Higashi H, Obuse C, Umeda M, Murata-Kamiya N, Saito Y, et al. Helicobacter pylori CagA targets PAR1/MARK kinase to disrupt epithelial cell polarity. Nature. 2007:447(7142):330-3.

39. Rios M, Foretz M, Viollet B, Prieto A, Fraga M, Garcia-Caballero T, et al. Lipoprotein internalisation induced by oncogenic AMPK activation is essential to maintain glioblastoma cell growth. Eur J Cancer. 2014:50(18):3187-97.

40. Nieto MA. Epithelial plasticity: a common theme in embryonic and cancer cells. Science. 2013:342(6159):1234850

41. Thiery JP. Epithelial-mesenchymal transitions in development and pathologies. Curr Opin Cell Biol. 2003;15(6):740-6.

42. Zeisberg M, Neilson EG. Biomarkers for epithelial-mesenchymal transitions. J Clin Invest. 2009;119(6):1429-37.

43. Perl AK, Wilgenbus P, Dahl U, Semb H, Christofori G. A causal role for E-cadherin in the transition from adenoma to carcinoma. Nature. 1998;392(6672):190-3.

44. Nieman MT, Prudoff RS, Johnson KR, Wheelock MJ. N-Cadherin promotes motility in human breast cancer cells regardless of their E-cadherin expression. J Cell Biol. 1999;147(3):631-43.

45. Hazan RB, Phillips GR, Qiao RF, Norton L, Aaronson SA. Exogenous expression of $\mathrm{N}$-cadherin in breast cancer cells induces cell migration, invasion, and metastasis. J Cell Biol. 2000;148(4):779-90.

46. Wheelock MJ, Johnson KR. Cadherins as modulators of cellular phenotype. Annu Rev Cell Dev Biol. 2003;19:207-35.

47. Bek S, Kemler R. Protein kinase CKII regulates the interaction of beta-catenin with alpha-catenin and its protein stability. J Cell Sci. 2002;115(24):4743-53.

48. Kim J, Yu W, Kovalski K, Ossowski L. Requirement for specific proteases in cancer cell intravasation as revealed by a novel semiquantitative PCR-based assay. Cell. 1998;94(3):353-62.

49. Rubinfeld B, Robbins P, El-Gamil M, Albert I, Porfiri E, Polakis P. Stabilization of beta-catenin by genetic defects in melanoma cell lines. Science. 1997;275(5307):1790-2.

50. Chaiyadet S, Smout M, Laha T, Sripa B, Loukas A, Sotillo J. Proteomic characterization of the internalization of Opisthorchis viverrini excretory/ secretory products in human cells. Parasitol Int. 2016;\$1383-5769(16)30007-1. 